\title{
Original Article \\ Reducing the number of sentinel nodes removed in melanoma patients: $A$ prospective study
}

\author{
Danino AM, Kadlub N, Dalac S*, Boichot C**, Malka G \\ Departments of Plastic Surgery, ${ }^{*}$ Dermatology and ${ }^{* *}$ Nuclear Medicine, Dijon University Hospital, Dijon \\ University, France
}

Correspondence to: Dr. Danino Alain, E-mail: alain.danino@chu-dijon.fr

\begin{abstract}
CONTEXT: Since 1992, sentinel lymph node (SLN) biopsy was generally applied to melanoma for tumor staging. As the literature points out, an increasing number of nodes are being removed for each procedure, driving up the cost for this procedure and wandering away from the defining concept of sentinel lymph node. AIMS: The objective of the current study was to show that the number of sentinel lymph node s removed can be minimized without influencing the reliability of tumor staging. MATERIALS AND METHODS: We conducted a single-arm prospective study in patients with stage I melanoma. For each patient, the sentinel lymph node was identified using the hand-held gamma probe technique. We removed only the hottest nodes as well as the nodes with radioactivity greater than $70 \%$ compared to the hottest. We analyzed the characteristics of each melanoma, the success rate of this procedure, how many nodes were removed and how many had micro metastases. STATISTICAL ANALYSIS: The results were compared to those of the literature, previously published Porter study using the chi-square test. RESULTS: We included 90 patients. The success rate of this technique was $100 \%$. We dissected 1.3 sentinel lymph nodes for each patient, with $22 \%$ positive SLN. Statistical analyses point out a better selectivity of our study for a similar rate of pathological positivity and recurrence compared to the literature. CONCLUSIONS: Our technique for decreasing the number of sentinel lymph nodes removed is reliable. The removal of minimal number of nodes doesn't compromise the sensitivity of tumor staging, while it does reduce the cost of the procedure.
\end{abstract}

Key words: Melanoma, nodal metastases, sentinel node

\section{Introduction}

Since Morton et al ${ }^{[1]}$ reported lymphatic mapping and sentinel lymphadenectomy, sentinel lymph node biopsy has become a widely accepted method of staging lymph nodes for patients with melanoma.

Morton et al initially used a tracer injection of blue dye to detect the sentinel lymph node (SLN). The addition of radioactive colloid has improved sentinel lymph node identification rates, but at the same time, it has also resulted in a greater number of identified sentinel lymph nodes per basin and patient. There is no standard definition of isotope mapping success. The derivation of the exact count level or ratio defining the sentinel lymph node has been largely empirical and arbitrary.

Current literature points out an increasing number of nodes removed for each procedure, leading to a higher human and economical cost for the procedure, which is considered to be experimental.

Our objective was to show that we can minimize the number of sentinel lymph nodes removed without compromising the reliability of tumor staging. 


\section{Materials and Methods}

A single-arm prospective study with an attempt to compare the data with the historical study of Porter et al was conducted between January 2002 and September 2004. A college of dermatologists and surgeons of our university selected the study of Porter et al for the details concerning the number of nodes removed.

Patients aged greater than 18 years with cutaneous melanoma of $\geq 1 \mathrm{~mm}$ Breslow thickness and clinically negative regional lymph nodes were eligible after providing informed consent.

\section{Lymphography}

On the day of lymph node dissection, an intradermal injection of $0.2 \mathrm{ml}(10-15 \mathrm{MBq})$ colloidal particles labeled with technetium $99 \mathrm{~m}$ was given.

The radiopharmaceutical was injected into both sides of the scar from the diagnostic excision. The technetium $99 \mathrm{~m}$ nanocolloid is transported through the lymphatic system and lodges in the SLNs, which can be visualized by dynamic and static views. The location of the SLN was marked on the skin with a pen.

\section{Surgical technique}

An excision of the melanoma is realized. The exact localization of the sentinel lymph node was determined preoperatively using a hand-held gamma counter. A small cutaneous incision was made over the area where the maximum radioactivity was detected. The most radioactive node and the nodes with radioactivity greater than $70 \%$ compared to the hottest node were dissected. The sentinel lymph node was removed and sent to the pathology lab. If metastatic disease was documented, a regional radical dissection was performed.

\section{Pathological examination}

Once the sentinel lymph node was removed, it was immediately sent to the pathologist. Three cuts from both surfaces were colored with Hematoxylin and Eosin $(\mathrm{H}$ and $\mathrm{E})$ and examined microscopically for metastatic localization. After preserving in formaldehyde, six sections were stained by an immunocytochemical method with anti-protein S100 serum, anti HMB45 and anti MelanA antibodies.

\section{Statistical evaluation}

We analyzed the characteristics of the melanoma, the success rate of the procedure, how many nodes were removed and how many had micrometastases. The results were compared to the results of the Porter's survey ${ }^{[2]}$ using the Chi-square test. Significance was defined as $P>0.05$.

\section{Results}

Overall, 90 patients were included in this study. The clinical pathologic characteristics of the study group are listed in Table 1. The mean age was 62.8 years (range, 25-90 years). The mean tumoral thickness was 2.96 (Range 0.3-20 mm).

The site of the primary melanoma was well distributed into all anatomical categories, including the trunk (14\%), the head and neck (14\%), the upper extremities (26\%) and the lower extremities (46\%). One lymph node basin per patient was mapped. Sentinel nodes were identified in $100 \%$ of cases. A total of 105 sentinel lymph nodes was identified (mean $=1.3$ per patient; range $1-3$ ). In

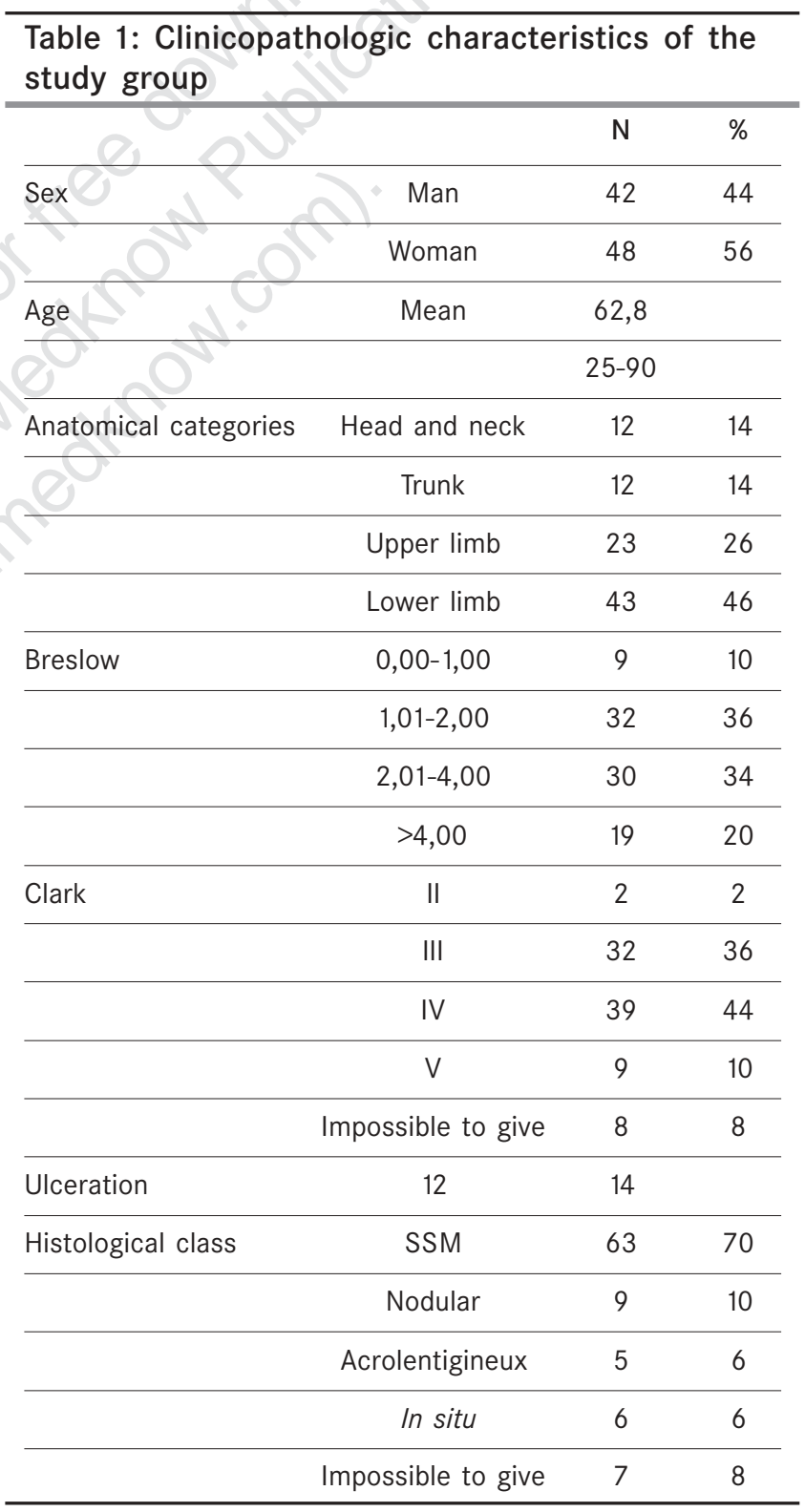


$74 \%$ of patients, only 1 lymph node was removed; in $22 \%, 2$ SLNs were identified; and in 3 patients (4\%), 3 SLNs were removed [Table 2].

Metastasized melanoma was detected in 19 SLNs from 19 patients $(22 \%)$. All of these patients went on to have a complete dissection of the involved basin and additional positive nodes were found in only 1 patient. During the follow-up (mean=18.8 months; range 8-32 months), one patient with negative sentinel lymph node had a basin recurrence $(2.5 \%)$, whereas two patients with positive sentinel lymph node had distant metastases (18.18\%).

These results were compared with the results of Porter's study, shown in Table 3 . There was no significant difference with respect to the success rate of the procedure, the detection of metastatic nodes and the recurrence rate after negative sentinel lymph node biopsy; however, we point out a significant difference with regard to the number of removed sentinel lymph nodes.

\begin{tabular}{lcc}
\hline $\begin{array}{l}\text { Table 2: Number of SLN removed for each } \\
\text { procedure }\end{array}$ & $\mathrm{N}$ & $\%$ \\
\hline Number of SLN removed & 66 & 74 \\
\hline 1 & 20 & 22 \\
\hline 2 & 4 & 4 \\
\hline 3 & 90 & 100 \\
\hline Total
\end{tabular}

\begin{tabular}{|c|c|c|c|c|}
\hline & & $\begin{array}{l}\text { Dijon's } \\
\text { study }\end{array}$ & $\begin{array}{c}\text { Porter et al, } \\
\text { GA surgery, } \\
\text { aug } 2000\end{array}$ & $P$ \\
\hline $\mathrm{N}$ & 90 & 633 & & \\
\hline Success rate (\%) & 100 & 99 & 1 & \\
\hline \multirow[t]{3}{*}{ Number of SLN removed } & 1 & 74 & 45 & $<0,05$ \\
\hline & 2 & 22 & 32 & \\
\hline & or $=3$ & 4 & 23 & \\
\hline SLN + (\%) & 22 & 18 & 0,5 & \\
\hline
\end{tabular}

\section{Recurrence after}

SLN - (\%)

$2,5 \quad 2,9$

\section{Discussion}

Before the introduction of sentinel lymph node biopsy, the watch-and-wait policy was generally applied to stage I melanoma. When lymph nodes became palpable, a lymph node dissection was performed. Previous findings recognized sentinel node status as an important prognostic factor. ${ }^{[3-5]}$ Patients with positive sentinel lymph node are more likely to develop local and distant metastases than those with negative sentinel lymph node biopsy. There is no proof that sentinel lymph node dissection improves survival of melanoma patients. The sentinel lymph node biopsy procedure is mainly used for staging of melanoma patients and to select patients for adjuvant therapies at an early stage. The addition of radioactive colloids has refined detection and has also resulted in a greater number of sentinel lymph nodes being identified. The first aim of our work was to show that a reduction of the number of nodes extracted is possible. In our study, we chose to use only radioactive detection in order to avoid allergic risk of blue. Multiple radioactive sentinel nodes were commonly detected in our survey; however, we removed only the most radioactive nodes (those with greater than $70 \%$ of the maximal detected radioactivity). When metastatic nodes were identified, the positive nodes were the most radioactive ones. Our results do not show any difference in the safety of the staging.

- In this study, $22 \%$ of patients with melanomas 1 $\mathrm{mm}$ or greater according to Breslow thickness were found to have histological evidence of nodal metastases, which is a higher rate than in other published studies. ${ }^{[6,7]}$

- Recurrence rate after histological SLN was 2.5\% in our survey; in literature studies, the recurrence rate in negative SLN is reported at $2-11 \%$. Recurrence after negative sentinel lymph node can be the result not only of procedure failure but also of transient metastases that can't be controlled by the surgeon. Recurrence can also be the result of a histological false negative result. When patients develop recurrent lymph node melanoma after SLN biopsy, reevaluation of SLN by tyrosinase and HMB 45 PCR often reveals micrometastases that were not appreciated at the time of the initial examination. ${ }^{[8]}$ Some false negative results are found in all studies.

\section{Conclusion}

We felt that sentinel lymph node could be identified 
using our technique of radioactive detection (dissection of the most radioactive node as well as the nodes with radioactivity greater than $70 \%$ compared to the hottest node), reducing the number of nodes removed. Removing more nodes than necessary increases the risks and cost of the sentinel lymph node procedure with no apparent impact on nodal staging.

\section{References}

1. Morton DL, Wen DR, Wong JH, Economou JS, Cagle LA, Storm FK, et al. Technical details of intraoperative lymphatic mapping for early melanoma. Arch Surg 1992;127:392-9.

2. Porter GA, Ross MI, Berman RS, Sumner WE $3^{\text {rd }}$, Lee JE, Mansfield $\mathrm{PF}$, et al. How many lymph nodes are enough during sentinel lymphadenectomy for primary melanoma? Surgery 2000; 128:306-11.

3. Cascinelli N, Belli F, Santimani M, Fait V, Testori A, Ruka W, et al. Sentinel Lymph Node biopsy in cutaneaous melanoma: The WHO melanoma program experience. Ann Surg Oncol
2000;7:469-74.

4. Jansen L, Nieweg OE, Peterse JL, Hoefnagel A, Valdes Olmos RA, Kroon BB. Reliability of sentinel lymph node biopsy for staging melanoma. Br J Surg 2000;87:484-9.

5. Gershenwald JE, Colome MI, Lee JE, Mansfield PF, Tseng C, Lee $\mathrm{JJ}$, et al. Patterns of recurrence following a negative sentinel lymph node biopsy in 243 patients with stage I or II melanoma. J Clin Oncol 1998; 16:2253-60.

6. Chao C, Wong SL, Ross MI, Reintgen DS, Noyes RD, Cerrito PB et al. Patterns of early recurrence after sentinel lymph node biopsy for melanoma. Am J Surg 2002; 18:520-4.

7. Kuo CT, Hoon DA, Takeuchi H, Turner R, Wang HJ, Morton DL, et al. Prediction of disease outcome in melanoma patients by molecular analysis of paraffin-Embedded sentinel Lymph Nodes. J Clin Oncol 2003;21:3566-72.

8. Ribuffo D, Gradilone A, Vonella M, Chiummariello S, Cigne E, Haliassos N, et al. Prognostic significance of reverse tanscriptase polymerase chain reaction-Negative sentinel nodes in malignant melanoma. Ann Surg Oncol 2003;10:396-402.

Source of Support: Nil, Conflict of Interest: None declared.

\section{Author Help: Sending a revised article}

1) Include the referees' remarks and point to point clarification to those remarks at the beginning in the revised article file itself. In addition, mark the changes as underlined or coloured text in the article. Please include in a single file
a. referees' comments
b. point to point clarifications on the comments
c. revised article with text highlighting the changes done

2) Include the original comments of the reviewers/editor with point to point reply at the beginning of the article in the 'Article File'. To ensure that the reviewer can assess the revised paper in timely fashion, please reply to the comments of the referees/editors in the following manner.

- There is no data on follow-up of these patients.

Authors' Reply: The follow up of patients have been included in the results section [Page 3, para 2]

- $\quad$ Authors should highlight the relation of complication to duration of diabetes.

Authors' Reply: The complications as seen in our study group has been included in the results section [Page 4, Table] 